\title{
Long solitary internal waves in stable stratifications
}

\author{
W. B. Zimmerman ${ }^{1}$ and J. M. Rees ${ }^{2}$ \\ ${ }^{1}$ Department of Chemical and Process Engineering, University of Sheffield, Sheffield S1 3JD, UK \\ ${ }^{2}$ Department of Applied Mathematics, University of Sheffield, Sheffield S3 7RH, UK
}

Received: 2 December 2003 - Accepted: 21 January 2004 - Published: 14 April 2004

\begin{abstract}
Observations of internal solitary waves over an antarctic ice shelf (Rees and Rottman, 1994) demonstrate that even large amplitude disturbances have wavelengths that are bounded by simple heuristic arguments following from the Scorer parameter based on linear theory for wave trapping. Classical weak nonlinear theories that have been applied to stable stratifications all begin with perturbations of simple long waves, with corrections for weak nonlinearity and dispersion resulting in nonlinear wave equations (Korteweg-deVries (KdV) or Benjamin-Davis-Ono) that admit localized propagating solutions. It is shown that these theories are apparently inappropriate when the Scorer parameter, which gives the lowest wavenumber that does not radiate vertically, is positive. In this paper, a new nonlinear evolution equation is derived for an arbitrary wave packet thus including one bounded below by the Scorer parameter. The new theory shows that solitary internal waves excited in high Richardson number waveguides are predicted to have a halfwidth inversely proportional to the Scorer parameter, in agreement with atmospheric observations. A localized analytic solution for the new wave equation is demonstrated, and its soliton-like properties are demonstrated by numerical simulation.
\end{abstract}

\section{Introduction}

Chemical engineering abounds with unit operations where solitary waves can be induced. Film flows, especially in condensers and distillation columns, give rise to sheared, stable stratifications where solitary waves can be induced by mass transfer (Hewakandamby and Zimmerman, 2003) or heat transfer effects that are driven by surface tension gradients. In this paper, motivated by recent studies of surfactant spreading on sheared, stable stratifications and observations in the stable Antarctic boundary layer (Rees and Rottman,

Correspondence to: $\mathrm{W}$. B. Zimmerman

(w.zimmerman@shef.ac.uk)
1994), we revisit the classical analysis leading to the conclusion that large amplitude waves can be induced in the infinity long limit. Scorer (1949) pointed out that longer waves than a particular value selected by the shear and stratification profiles, radiate vertically, and thus are not trapped by the waveguide. This condition is the modification due to shear of the classical Brunt-Väisälä frequencyfor vertical radiation of waves in a purely stratified fluid. In this paper, we take into account this limitation and pose a derivation of the nonlinear evolution equation (NEE) which reduces to the classical Korteweg-de Vries equation in the case that the Scorer wavenumber approaches zero so that infinitely long waves are trapped.

Benney (1966) and Benjamin (1966) independently derived the first NEE for large amplitude solitary disturbances in a stable stratification with shear flow. They both assumed weak nonlinearity and weak dispersion due to a long wave approximation. The derived NEE, which applies equally to either temperature or streamfunction disturbances, takes the form of a Korteweg-deVries (KdV) equation, with coefficients that depend on both the conditions of the waveguide (functionals of the stratification and shear profiles) and on the characteristics of the disturbance itself (wavelength and amplitude):

$$
\frac{\partial A}{\partial t}+(c+A) \frac{\partial A}{\partial x}+\sigma \frac{\partial^{3} A}{\partial x^{3}}=0 .
$$

This has the solution that the amplitude $A$, at time $t$ and position $x$, is

$A=3 A_{0} \operatorname{sech}^{2}\left(\left(\frac{A_{0}}{4 \sigma}\right)^{1 / 2}\left(x-\left(c+A_{0}\right) t\right)\right)$

where $A_{0}$ is the amplitude of the initial disturbance, $c$ the phase velocity of infinitely long waves, and $\sigma=c H^{2} / 6$ is the parameter measuring dispersion. $H$ is the layer depth. The halfwidth of the solitary wave is $\left(A_{0} / 16 \sigma\right)^{-1 / 2}$. Because this halfwidth is inversely proportional to $\sqrt{\sigma}$, it follows that the wavelength of a localised disturbance depends inversely on 
the spectral width of the wave packet that characterizes the Fourier power spectrum of the disturbance.

The KdV theory proposed by Benney and Benjamin is robust, with many applications in atmospheric and oceanographic analysis, e.g. Doviak et al. (1991). Nevertheless, there is a fundamental problem with the derivation of the $\mathrm{KdV}$ theory from the infinitely long wave limit. Essentially, in some circumstances, the waveguide will be depleted of wave energy in long scales. This is described more fully below. Benney's derivation begins with a linear wave equation that to leading order is a simple wave of infinitely long wavelength, satisfying:

$$
\frac{\partial A}{\partial t}+c \frac{\partial A}{\partial x}=0
$$

Scorer (1949), however, considers modified Brunt-Väisälä vertical buoyancy waves in the presence of shear flow. He concludes that wave energy is only trapped if it is confined to wavenumbers $k$ that are greater than the Scorer parameter $\mathcal{L}$ defined here:

$\mathcal{L}^{2}=\frac{N^{2}}{(\hat{u}-v)^{2}}-\frac{1}{(\hat{u}-v)} \frac{d^{2} \hat{u}}{d y^{2}}$

$N$ is the Brunt-Väisälä frequency, $v$ is the phase velocity, and $\hat{u}(y)$ is the background shear profile, which is assumed steady before and after the passage of the disturbance. Wave energy can radiate vertically out of the waveguide as Brunt-Väisälä waves if the wavenumber of the disturbance is smaller than $\mathcal{L}$. Scorer (1949), showed that the Fourier component of the vertical velocity $\hat{w}_{k}$ satisfies in the linear approximation:

$\frac{d^{2} \hat{w}_{k}}{d y^{2}}+\left(\mathcal{L}^{2}-k^{2}\right) \hat{w}_{k}=0$.

$y$ is the vertical coordinate and $k$ is the wavenumber of the disturbance. Scorer showed that when $\mathcal{L}^{2}$ decreases with height, energy can be trapped at low levels. More generally, within in any waveguide, if $\mathcal{L}^{2}>k^{2}$, Eq. (5) admits oscillatory solutions that radiate vertically as Brunt-Väisälä waves. When $\mathcal{L}^{2}<k^{2}$, one solution of Eq. (5) is a bound-state, i.e. energy is trapped in the waveguide and can only propagate within the waveguide. The other solution to Eq. (5) in this case is exponential growth far away from the waveguide, which is rejected as aphysical.

The wavelength of solitary disturbances over an Antarctic ice shelf has been discussed by Rees and Rottman (1994). These authors provided empirical evidence showing that even large amplitude disturbances have halfwidths that agree remarkably well with the linear Taylor-Goldstein theory described by Scorer (1949). This is a surprising result in that for the large amplitude disturbances that were reported by Rees and Rottman (1994), nonlinear effects are important and linear theory would be expected to fail. They cited Scorer's argument that linear waves longer than wavenumber $\mathcal{L}$ would radiate vertically out of the waveguide. Since wave energy at scales shorter than the Scorer wavenumber $\mathcal{L}$ are trapped in the waveguide, it is not at first clear why large amplitude solitary disturbances exhibit the Scorer wavenumber.

Ursell (1953) resolved a long standing paradox in the theory of solitary surface gravity waves by coining a new dimensionless number $\mathrm{Ur}=A_{0} L^{2} / H^{3}=\varepsilon / \mu^{2}$, the ratio of two dimensionless numbers. $\varepsilon=A_{0} / H$ is the ratio of the maximum streamfunction displacement to the height $H$ of the waveguide. $\mu=H / L$ is the ratio of the height of the waveguide to the expected wavelength $L$ of the long wave disturbance. Thus, $\varepsilon$ and $\mu$ are properties of the resultant wave generated from some initial disturbance to the waveguide. Since the formation of a solitary wave may occur after a combination of the nonlinear breaking of the initial disturbance or the dispersion of different components, the length scales of the initial conditions may have little bearing on the resultant wave. Thus, the description of solitary wave motion by the parameters $\mu$ and $\varepsilon$ ignores the history of its formation. Ursell suggested that when $\mathrm{Ur} \sim 1$, with both $\varepsilon \ll 1$ and $\mu \ll 1$, solitary waves of permanent form can propagate. In fact, this argument previously appeared in the pioneering papers of (Boussinesq, 1871a,b, 1872) and was understood earlier by Lord Rayleigh (Rayleigh, 1876). It should be noted, that in defining $\mu$ and $\varepsilon$ as above, the solitary wave is presupposed to exist. On dimensional analysis grounds, $\mu$ and $\varepsilon$ are independent parameters. The Ursell hypothesis relating the two follows from the stronger constraint that the solitary wave should have permanent form. The specific functional relationship that Ursell proposed was physically motivated by the mechanism maintaining permanent form-nonlinear steepening balancing wave dispersion.

Internal solitary waves may propagate in a waveguide of a stratified fluid layer, with and without shear. However, the inclusion of shear introduces a free parameter, the Richardson number $R i$, the ratio of buoyancy to shear forces, which affects the balance of nonlinearity and dispersion in wave pro- pagation. Dimensional analysis, along with the constraint that the waveform be permanent, results in the functional relationship $\varepsilon=f(\mu, R i)$. The form of this functional relationship should be based on accounting for the relative strength of shear and stratification in maintaining the balance between nonlinearity and dispersion in the propagation of a wave of permanent form. It follows that the wavelength that should be selected in the long time asymptotic state should be that with weakest dispersion balancing weak nonlinearity - so that neither mechanism is strong enough to break up the permanent form. Thus, in the time asymptotic state, the wavelength should be as long as possible. The longest wavelength admissible, therefore, in a sheared, stratified flow, is identified with the Scorer wavenumber $\mathcal{L}$.

Benney (1966) and Benjamin (1966) independently treated the weakly nonlinear shallow layer approximation, showing that disturbances travel according to the KortewegdeVries equation if the bounding surfaces of the layer are material surfaces (no flux of momentum vertically, no horizontal stress). This assumption is not always compatible with a continuously stratified fluid where a small stratum forms the waveguide, since waves longer than the Scorer wavenumber 

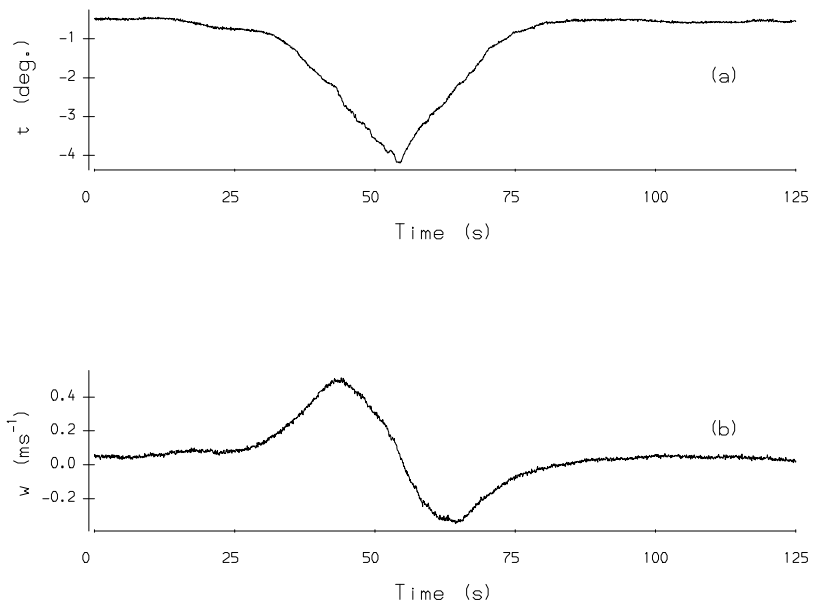

Fig. 1. Temperature (re-based) and vertical velocity of a solitary disturbance observed at Halley Station by the British Antarctic Survey on 6 June 1986 at a height of $16 \mathrm{~m}$. Measurements were made using a sonic anemometer and were recorded at $20 \mathrm{~Hz}$.

$\mathcal{L}$ radiate vertically. From the definition (4), there are two distinct cases: $\mathcal{L}^{2}>0$, where longer waves radiate vertically; $\mathcal{L}^{2}<0$, where all wavenumbers are trapped. In this latter case, the lack of radiation vertically is consistent with the boundary conditions of the model adopted by Benney (1966) and Benjamin (1966) leading to the KdV theory-parallel, impenetrable stress-free surfaces. Although in this paper the focus is on wave propagation with $\mathcal{L}^{2}>0$, observations of both cases in the STABLE data sets of the British Antarctic Survey are reported.

When $\mathcal{L}^{2}>0$, from the analysis of Scorer, it is clear that the $k=0$ long wave limit is inaccessible when shear and stratification establish a waveguide that is one stratum of the deeper fluid, since all wavenumbers smaller than $|\mathcal{L}|$ will radiate vertically. Thus, long times after the initial disturbance show depletion in the horizontal waveguide of energy in the $k \in[0,|\mathcal{L}|)$ band. However, for wavenumbers greater than $|\mathcal{L}|$, the upper and lower surfaces of waveguide do act as material surfaces, since these wavenumbers are trapped. It follows that the proper nonlinearisation of the linear dispersive waves is bounded below in wavenumber space by $|\mathcal{L}|$, and is, therefore, distinctly different from the analysis of Benney (1966) and Benjamin (1966), which start from infinitely long waves in the leading order separation condition (3). In the theory that follows, the basic disturbance is taken to be a harmonic wave with wavenumber $k^{2}>\mathcal{L}^{2}$, and the first solvability condition leads to an NEE similar in form to the $\mathrm{KdV}$ equation.

The paper is organised as follows: In Sect. 2, two wave events with $\mathcal{L}^{2}$ of different signs are reported from the British Antarctic Survey STABLE data sets (King and Anderson, 1988). In Sect. 3, a KdV-like theory for arbitrary wavelength basic disturbances is derived. When $\mathcal{L}^{2}>0$, the theory is only applicable for nearly monochromatic disturbances. For $\mathcal{L}^{2}<0$, the trapping in the waveguide is complete and the theory is generally applicable. Section 4 discusses the re-
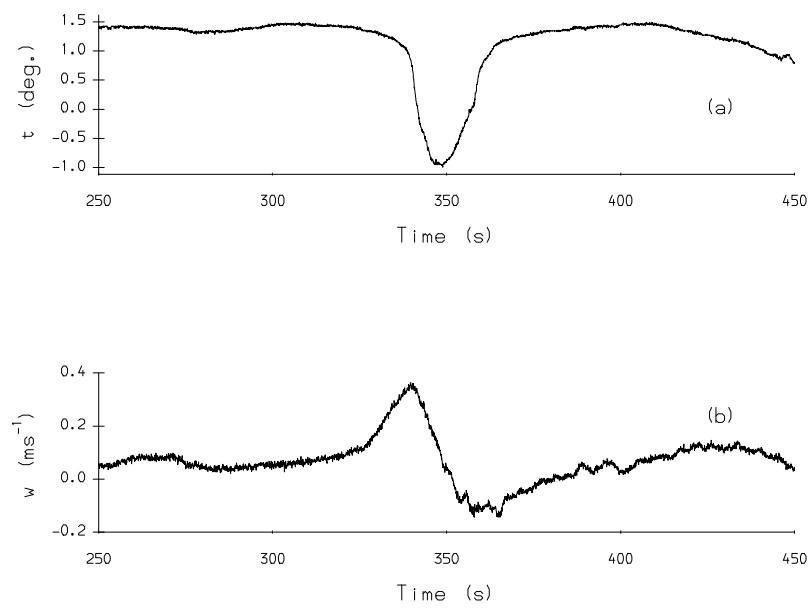

Fig. 2. Temperature (re-based) and vertical velocity of a solitary disturbance observed at Halley Station by the British Antarctic Survey on 14 August 1986.

sults of the theory, demonstrating an analytic solution to the full NEE. Furthermore, the prediction that the halfwidth of the solitary wave by the Scorer parameter, which is demonstrated in Sect. 2 on the STABLE data set, holds theoretically in large $R i$ waveguides. Section 6 explores numerical simulations of the NEE, which provide some evidence of solitonic behaviour for the dynamics of overtaking collisions involving solitary wave solutions to the NEE. In Sect. 6, conclusions are drawn.

\section{Observations of atmospheric solitary disturbances in the Antarctic}

As a result of strong radiative cooling at the earth's surface during the Antarctic winter, a strong, long-lasting, stably stratified atmospheric boundary layer is established at Halley IV station, Antarctica $\left(75.6^{\circ} \mathrm{S}, 26.7^{\circ} \mathrm{W}\right)$. Halley IV station is situated on the Brunt Ice Shelf which provides a uniform fetch of $10-40 \mathrm{~km}$ in all directions. Thus the site serves as an "ideal" natural laboratory for the study of the stable atmospheric boundary layer and any wave-like structures it may support. The British Antarctic Survey have compiled a database of meteorological measurements from Halley IV station during their STABLE project for the purpose of developing a climatology of gravity wave activity in the boundary layer (King and Anderson, 1988; Rees, 1991; Rees et al., 2000). The solitary large amplitude disturbances reported here are typical examples of several observations in the data set. The meteorological data recorded in the 1986 STABLE project included wind speed, wind direction, and temperature on a $32 \mathrm{~m}$ mast, with measurements at six heights.

Figure 1 shows the temperature fluctuation about an arbitrary mean and vertical velocity of a wave event recorded 6 June 1986 at a height of $16 \mathrm{~m}$. By eye, it is apparent that the fundamental assumption of both the KdV theory and BDO 

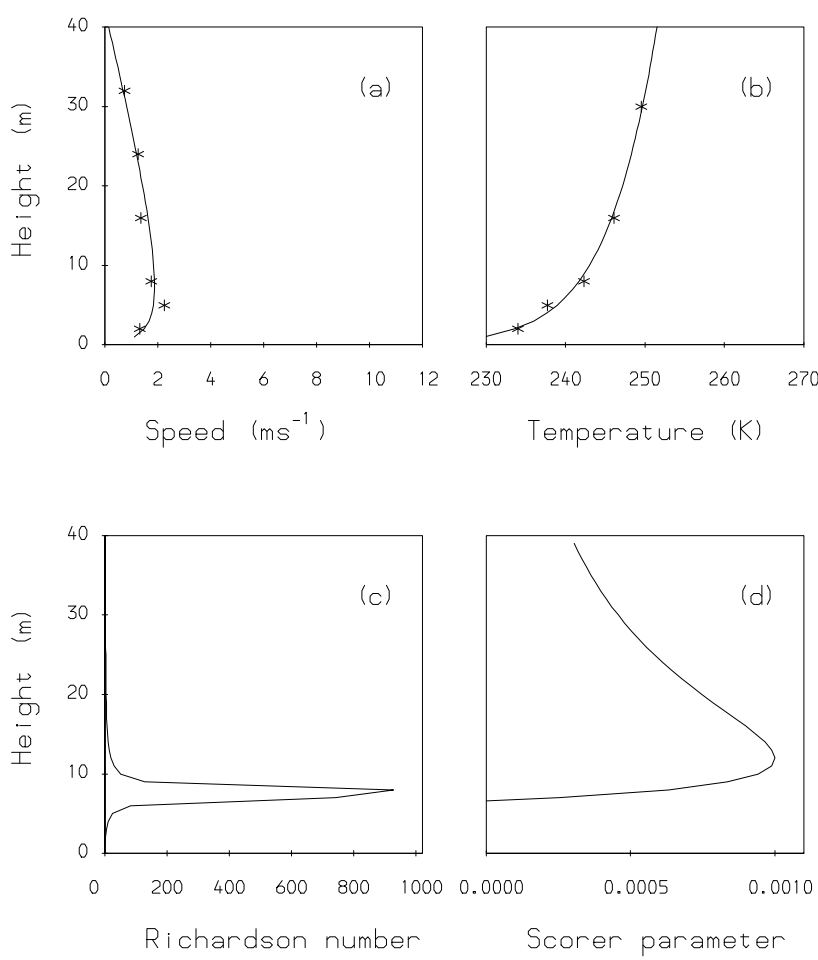

Fig. 3. Computed shear profile, temperature, Richardson number and Scorer parameter of the observed solitary disturbance at Halley Station by the British Antarctic Survey on 6 June 1986, at $100 \mathrm{~s}$ (after the wave passed) with the origin of time defined in Fig. 2, following Rees and Rottman.

theory is approximately satisfied, i.e. that the streamfunction and temperature field $\mathcal{T}$ have the same disturbance waveform in the horizontal waveguide. Since vertical velocity $w$ is the expressed through a spatial derivative of the streamfunction, this can be modelled by separation of variables:

$\mathcal{T}=A(x, t) \theta(y)+O(\varepsilon)$

$w=\frac{\partial A}{\partial x} \phi(y)+O(\varepsilon)$

For simple long waves, to a first approximation

$\frac{\partial A}{\partial x}=-\frac{1}{c} \frac{\partial A}{\partial t}$

Since it appears that the vertical velocity in Fig. 1 is roughly proportional to the negative time derivative of the disturbance temperature field, it is consistent with the premise that either weakly nonlinear theory ( $\mathrm{KdV}$ or BDO) applies.

Figure 2 is from a different wave event which occurred on 14 August 1986. Although the qualitative picture remains very similar to the event on 6 June 1986, there is a fundamental difference in the vertical structure of the waveguides. Figure 3 shows fitted profiles for the background shear flow and temperature, with the Richardson number and Scorer parameter computed from them. The details of the modelling of the data were presented by Rees and Rottman (1994). What is apparent from the Richardson number plot is that there is
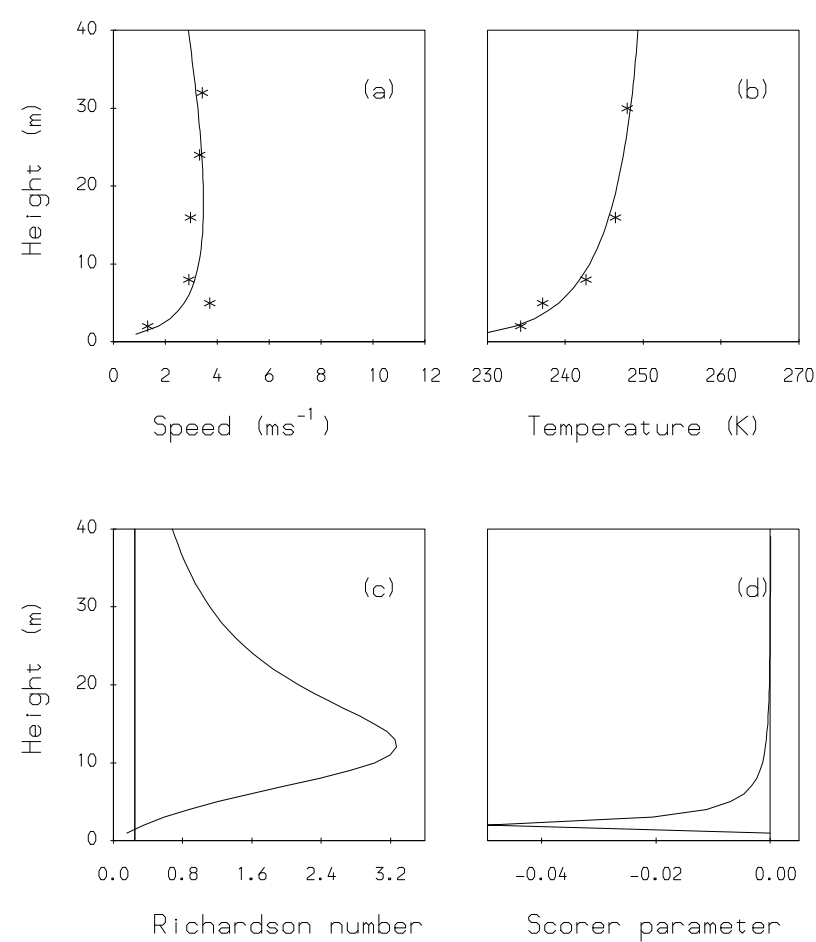

Fig. 4. Computed speed, temperature, Richardson number and Scorer parameter of the observed solitary disturbance at Halley Station by the British Antarctic Survey on 14 August 1986, at $300 \mathrm{~s}$ (after the wave passed) with the origin of time defined in Fig. 3, following Rees and Rottman.

a stratum of very strong stratification at a low level (circa $10 \mathrm{~m}$ ) subtended by upper and lower regions of strong shear. In the vicinity of this stratum, the Scorer parameter has an internal maximum which is greater than zero, the condition under which Scorer argued that energy could be trapped at higher wavenumbers at low levels. Those authors noted that for this event, the wavelength of waves at the Scorer cut-off is approximately:

$\frac{2 \pi}{\sqrt{0.001 \mathrm{~m}^{-2}}} \approx 200 \mathrm{~m}$

Rough statistics on the solitary disturbance give duration $50 \mathrm{~s}$ and phase velocity $4.5 \mathrm{~m} / \mathrm{s}$, i.e. a wavelength of $225 \mathrm{~m}$. Given the accuracy at which the duration can be estimated, say between $40 \mathrm{~s}$ (wavelength $180 \mathrm{~m}$ ) and $50 \mathrm{~s}$, this is quite astonishing agreement. Nor is this an isolated event. The Scorer parameter is negative below $5 \mathrm{~m}$, indicating that all wavenumbers $k \geq 0$ are trapped below this height.

Figure 4 gives the equivalent observed and derived features for the wave event on 14 August 1986. In this instance, the stratum for which stratification dominates shear is both broader and weaker. The Scorer parameter is negative in this stratum, indicating that all wavenumbers $k \geq 0$ are trapped. Estimates of a duration of $33 \mathrm{~s}$ and phase velocity of $7.0 \mathrm{~m} / \mathrm{s}$ give a similar wavelength of $210 \mathrm{~m}$.

The events reported here lead to the question of whether there exists a selection mechanism for the wavelength of 
solitary internal waves. The focus on the Scorer parameter, given the indication that when $\mathcal{L}^{2}>0$, there is apparent correlation with observed wavelengths, begs the question of the mechanism of wavelength selection. Further, if there is a wavelength selection for waveguides with $\mathcal{L}^{2}>0$, is there none for $\mathcal{L}^{2}<0$ ? The theory developed in the next section is motivated by these questions.

\section{Model system}

\subsection{Equations and scaling}

Consider a fluid layer bounded above and below by parallel material surfaces. The upper material surface is constrained to move at velocity $U=\gamma H$, where $\gamma$ is the shear rate, and is held at constant temperature $\mathcal{T}=1$. The lower is held at $\mathcal{T}=0$ and at rest. As the model that is proposed is Galilean invariant, an arbitrary uniform wind component could be included without loss of generality. The fluid is inviscid and nonconducting. All lengths are scaled by $H$, velocity by $U$, and time by $H / U$. The flow is assumed to be twodimensional and divergence free, thus expressible through a streamfunction, $(u, v)=\left(\Psi_{y},-\Psi_{x}\right)$. Scalar transport is modelled as convection only:

$\mathcal{T}_{t}+\Psi_{y} \mathcal{T}_{x}-\Psi_{x} \mathcal{T}_{y}=0$

A Boussinesq fluid is assumed, with density linearly related to temperature. The dimensionless density is $\rho=1-\beta \mathcal{T}$, where $\beta$ is the dimensionless group formed from the coefficient of thermal expansion $\beta^{*}$, the unscaled temperature difference between the upper and lower surfaces $\Delta \mathcal{T}$, and $\rho_{0}$, the density of the fluid at the reference temperature of the lower surface. Namely, $\beta=\beta^{*} \Delta \mathcal{T} / \rho_{0}$.

Vorticity $\omega=-\nabla^{2} \Psi$ is transported by

$$
\begin{aligned}
0=R i \mathcal{T}_{x} & +\left[\Psi_{y t}+\Psi_{y} \Psi_{x y}-\Psi_{x} \Psi_{y y}\right]_{y} \\
& +\left[\Psi_{x t}+\Psi_{y} \Psi_{x x}-\Psi_{x} \Psi_{x y}\right]_{x}
\end{aligned}
$$

which is the curl of the Boussinesq momentum equation. The Richardson number is a measure of the relative importance of buoyant forces to shear forces, $R i=\beta g H / U^{2}=N^{2} / \gamma^{2}$. The assumption of a Boussinesq fluid is that the velocity field is divergence-free, $\beta \equiv 0$, and the $R i$ remains finite. No penetration (and stress free) and fixed temperature boundary conditions are imposed:

$\left.\Psi\right|_{y=0}=\left.\mathcal{T}\right|_{y=0}=0$

$\left.\Psi\right|_{y=1}=\left.\mathcal{T}\right|_{y=1}=1$

Because there is no dynamic boundary condition, pressure was eliminated, resulting in the momentum (9).

\subsection{Wave disturbances of permanent form}

Generally, the flow and temperature fields can be arbitrarily decomposed into background fields and disturbance fields. To make the procedure deterministic, the assumption is made that the disturbance field has zero mean in some sense. The sense adopted here is that the background fields are timeaveraged and thus steady. Further, the background fields are prescribed and independent of the horizontal $x$-coordinate.

$\mathcal{T}=\hat{T}(y)+\varepsilon T(x, y, t)$

$\Psi=\hat{\psi}(y)+\varepsilon \psi(x, y, t)$

$\varepsilon=A_{0} / h$ is the dimensionless characteristic size of the disturbance fields, where $A_{0}$ is the maximum value of the initial disturbance. For convenience, the following representative case of linear shear and stratification are considered:

$\hat{T}=y$

$\hat{\psi}=\frac{y^{2}}{2}$

Since it is the long time waveform which is required, and it is expected that that the form of solution will be steady in a the frame of reference of the phase velocity $v$, the following coordinate transform is made:

$\left(\begin{array}{l}x \\ t\end{array}\right) \rightarrow\left(\begin{array}{c}\xi=x-v t \\ \tau=\varepsilon t\end{array}\right) ; \quad\left(\begin{array}{c}\frac{\partial}{\partial x}=\frac{\partial}{\partial \xi} \\ \frac{\partial}{\partial t}=\varepsilon \frac{\partial}{\partial \tau}-v \frac{\partial}{\partial \xi}\end{array}\right)$

It is anticipated that the approach to a wave of permanent form occurs over a slow time scale. Substituting Eq. (11) into Eqs. (8) and (9) yields

$$
\begin{aligned}
& T_{\xi}(y-v)-\psi_{\xi}=\varepsilon\left(-\theta_{\tau}+\psi_{\xi} T_{y}-\psi_{y} T_{\xi}\right) \\
& {\left[\psi_{y \xi}(y-v)-\psi_{\xi}\right]_{y}+R i T_{\xi}+\left[\psi_{\xi \xi}(y-v)\right]_{\xi}} \\
& \quad=\varepsilon\left(-\psi_{y \tau}+\left[\psi_{\xi} \psi_{y y}-\psi_{y} \psi_{\xi y}\right]_{y}-\psi_{\xi \xi \tau}\right) \\
& \quad+\varepsilon\left(\left[\psi_{\xi} \psi_{\xi y}-\psi_{y} \psi_{\xi \xi}\right]_{\xi}\right)
\end{aligned}
$$

If $\varepsilon \ll 1$, it is quite natural to consider the vanishing amplitude limit of $\varepsilon=0$. The basic disturbance $T^{(0)}, \psi^{(0)}$ satisfies

$$
\begin{aligned}
& T_{\xi}^{(0)}(y-v)-\psi_{\xi}^{(0)}=0 \\
& {\left[\psi_{y \xi}^{(0)}(y-v)-\psi_{\xi}^{(0)}\right]_{y}+\operatorname{Ri} T_{\xi}^{(0)}} \\
& +\left[\psi_{\xi \xi}^{(0)}(y-v)\right]_{\xi}=0
\end{aligned}
$$

The expectation that the disturbance should be wavelike in the horizontal at any given height $y$ is equivalent to the separation of variables

$T^{(0)}(\xi, y)=A(\xi) \theta(y)$
$\psi^{(0)}(\xi, y)=A(\xi) \phi(y)$

The system (15) then simplifies to

$$
\begin{aligned}
& A_{\xi}(\theta(y-v)-\phi)=0 \\
& A_{\xi}\left[\phi_{y}(y-v)-\phi\right]_{y}+\operatorname{Ri\theta } A_{\xi}+A_{\xi \xi \xi}(y-v) \phi=0
\end{aligned}
$$

The system (17) has solutions only if a separation condition on $A$ is satisfied, namely

$$
A_{\xi \xi \xi}=-k^{2} A_{\xi}
$$


The separation constant was chosen to clarify that $A$ must be a harmonic wave to leading order. If Eq. (18) holds, then the system (17) reduces to

$\theta=\frac{\phi}{y-v}$

$\left[\phi_{y}(y-v)-\phi\right]_{y}-k^{2}(y-v) \phi+R i \frac{\phi}{y-v}=0$

The second equation above is the well known TaylorGoldstein equation (Taylor, 1931; Goldstein, 1931) for the particular case of a constant buoyancy frequency. For prescribed $R i$, Eq. (19) is a two point boundary value problem $\left(\left.\phi\right|_{y=0}=\left.\phi\right|_{y=1}=0\right)$ for the eigenvalue $\nu$. In the long wave limit, the regular spectrum (Davey and Reid, 1977) of $v_{n}$, $n= \pm 1, \pm 2, \ldots$, and the singular spectrum (Maslowe and Redekopp, 1980) $\left(v_{n} \in[0,1]\right)$ are known when $R i>1 / 4$. For $0<R i<1 / 4, v_{n}$ are complex conjugates, implying that one harmonic wave grows without bound and the other decays.

Zimmerman and Velarde (1997) demonstrate an analytic solution for the higher order dispersive terms in the long wave expansion and solvability condition, which is equivalent to the series solution to Eq. (19) in powers of $k^{2}$ :

$v=c+\sum_{l=1}^{\infty} k^{2 l} s_{l}$

The first five $s_{l}$ were given in closed form for the regular modes. It was shown that the radius of convergence of the series $s_{l}$ is at least $k_{r}=1$ for all $R i$ and $k_{r} \approx 10$ for $R i \gg 1$. In general, with the exception of $R i \downarrow 1 / 4, c \gg s_{1} \gg s_{2} \gg$ etc., showing that phase velocity is highly insensitive to finite wavenumber effects.

\subsection{Perturbation expansion}

The basic wave solution (18) and the accompanying boundary value problem for the vertical modal structure Eq. (19) differ fundamentally from the basic state adopted in the shallow layer model of Benney (1966). His basic wave is a simple plane wave, taken in the infinitely long wave limit $k=0$, i.e. $A_{t}+c A_{x}=0$, where $v=c$ is the phase velocity found as an eigenvalue of Eq. (19) when $k=0$. Benney proceeded to expand in small $\varepsilon$ and $k^{2}$. There are good reasons to adopt the infinitely long wave as the basis of an expansion procedure: (i) there are closed form solutions for some shear profiles (Maslowe and Redekopp, 1980) and stratifications (Weidman and Velarde, 1992); (ii) the procedure shows that long wave disturbances approximately satisfy the KdV equation and thus have soliton or conoidal character. However, extending the analysis of Benney to a viscous fluid is problematic-awkward questions about the ordering of weak nonlinearity, weak dispersion, and weak dissipation arise. The long wave limit presupposes that viscosity can only have a damping effect on waves.

Here a regular perturbation expansion is adopted. The starting point is the separation condition (18), which permits separation of the full system (14) only up to errors of $O(\varepsilon)$.

$$
A_{\xi \xi \xi}=-k^{2} A_{\xi}+O(\varepsilon)
$$

The Ansatz that the full system separates with errors of $O\left(\varepsilon^{2}\right)$ is made with the error in Eq. (21) taking the explicit form

$A_{\xi \xi \xi}=-k^{2} A_{\xi}-\varepsilon \sum_{i} \lambda_{i} D^{(i)}(\xi)$

The unknown functions $D^{(i)}$ and coefficients $\lambda_{i}$ are determined by requiring that the $O(\varepsilon)$ equations have solutions. The temperature and streamfunction are approximated by

$$
\begin{aligned}
& T(\xi, y)=A(\xi) \theta(y)+\varepsilon T^{(1)}(\xi, y)+O\left(\varepsilon^{2}\right) \\
& \psi(\xi, y)=A(\xi) \phi(y)+\varepsilon \psi^{(1)}(\xi, y)+O\left(\varepsilon^{2}\right)
\end{aligned}
$$

The system which must be satisfied at $O(\varepsilon)$ is

$$
\begin{aligned}
& T_{\xi}^{(1)}(y-v)-\psi_{\xi}^{(1)}=A A_{\xi}\left(\phi \theta_{y}-\phi_{y} \theta\right)-A_{\tau} \theta \\
& {\left[\psi_{y \xi}^{(1)}(y-v)-\psi_{\xi}^{(1)}\right]_{y}+R i T_{\xi}^{(1)}+\psi_{\xi \xi \xi}^{(1)}(y-v)} \\
& \quad=A A_{\xi}\left[\phi \phi_{y y}-\phi_{y} \phi_{y}\right]_{y}+\phi \phi_{y}\left[A_{\xi}^{2}-A A_{\xi \xi}\right]_{\xi} \\
& \quad+-A_{\tau} \phi_{y}-A_{\xi \xi \tau} \phi+\sum_{i} \lambda_{i} D^{(i)} \phi(y-v)
\end{aligned}
$$

The above system separates, by analogy with Eq. (16)

$\begin{aligned} T^{(1)} & =\sum_{i} F^{(i)}(\xi) \theta^{(i)}(y) \\ \psi^{(1)} & =\sum_{i} F^{(i)}(\xi) \phi^{(i)}(y)\end{aligned}$

provided $F_{\xi}^{(i)}=D^{(i)}$ and to a first approximation the $F^{(i)}$ are harmonic waves

$F_{\xi \xi \xi}^{(i)}=-\kappa^{2} F_{\xi}^{(i)}+O(\varepsilon)$

Linearity of Eq. (24) permits the solution to be constructed by the superposition of the solutions of five inhomogeneous boundary values problems, resulting from the assignment of the $D^{(i)}$ so that each component separates.

$$
\begin{aligned}
& D^{(1)}=A_{\tau} \\
& D^{(2)}=A_{\xi \xi \tau} \\
& D^{(3)}=A A_{\xi} \\
& D^{(4)}=A A_{\xi \xi \xi} \\
& D^{(5)}=A_{\xi} A_{\xi \xi}
\end{aligned}
$$

In the case of the linear terms $A_{\tau}$ and $A_{\xi \xi \tau}, \kappa=k$. For the nonlinear terms $A A_{\xi}, A A_{\xi \xi \xi}$ and $A_{\xi} A_{\xi \xi}, \kappa=2 k$. The five 
inhomogeneous systems that arise are

$M_{k}\left[\begin{array}{l}\theta^{(1)} \\ \phi^{(1)}\end{array}\right]=\left[\begin{array}{c}-\theta \\ \phi_{y}-\lambda_{1}(y-v) \phi\end{array}\right]$

$M_{k}\left[\begin{array}{l}\theta^{(2)} \\ \phi^{(2)}\end{array}\right]=\left[\begin{array}{c}\theta \\ \phi_{y}-\lambda_{2}(y-v) \phi\end{array}\right]$

$M_{2 k}\left[\begin{array}{l}\theta^{(3)} \\ \phi^{(3)}\end{array}\right]=\left[\begin{array}{c}\theta_{y} \phi-\theta \phi_{y} \\ {\left[\phi \phi_{y y}-\phi_{y}^{2}\right]_{y}-\lambda_{3}(y-v) \phi}\end{array}\right]$

$M_{2 k}\left[\begin{array}{l}\theta^{(4)} \\ \phi^{(4)}\end{array}\right]=\left[\begin{array}{c}0 \\ -\phi \phi_{y}-\lambda_{4}(y-v) \phi\end{array}\right]$

$M_{2 k}\left[\begin{array}{l}\theta^{(5)} \\ \phi^{(5)}\end{array}\right]=\left[\begin{array}{c}0 \\ \phi \phi_{y}-\lambda_{5}(y-v) \phi\end{array}\right]$

The linear operator $M$ is identified by rewriting Eq. (19) as a homogeneous boundary value problem. The Fredholm alternative theorem provides a criterion for the existence of solutions of the above five inhomogeneous boundary value problems-the inner product of the forcing vector with any eigenfunctions of the adjoint operator $M^{*}$ must vanish. Due to the extended separation of variables (22) and (25), the Fredholm alternative theorem holds independently for all $D^{(i)}$ since a linear system (28) results for each undetermined function $D^{(i)}$ that is solvable only if the identifications (27) are made. Thus since the quadratic order operators are all $M_{2 k}$, the criteria is that the inhomogeneous forcing vector must be orthogonal in these cases to the eigenfunctions of this operator $M_{2 k}$, not $M_{k}$. This solvability condition only obtains because the extended separation of variables (22) and (25) results in a hierarching of linear systems for which the superposition principle holds. This distinction must be made since there is no general solvability condition for fully nonlinear systems, for instance Eq. (14). The purpose of our analysis is to use perturbation methods and a separation scheme so that the component linear systems (28) are solvable.

In order to save on computations, it is desirable to work with a self-adjoint system. Following Weidman and Velarde (1992), it is observed that the boundary value problems for the modified vertical eigenfunctions

$\varphi^{(i)}=\frac{\phi^{(i)}}{y-v}$

are self-adjoint. Namely, the linear operator, on eliminating the temperatures $\theta^{(i)}$ is

$L_{k}[\varphi]=\left[(y-v)^{2} \varphi_{y}\right]_{y}+\left(R i-k^{2}(y-v)^{2}\right) \varphi$

The five inhomogeneous boundary value problems now for the $\varphi^{(i)}$ are

$$
\begin{aligned}
L_{k}\left[\varphi^{(1)}\right] & =\operatorname{Ri\theta }+\phi_{y}(y-v)-\lambda_{1}(y-v)^{2} \phi \\
L_{k}\left[\varphi^{(2)}\right] & =\phi(y-v)-\lambda_{2}(y-v)^{2} \phi \\
L_{2 k}\left[\varphi^{(3)}\right] & =-\operatorname{Ri}\left(\theta_{y} \phi-\theta \phi_{y}\right) \\
& +(y-v)\left[\phi \phi_{y y}-\phi_{y}^{2}\right]_{y}-\lambda_{3}(y-v)^{2} \phi \\
L_{2 k}\left[\varphi^{(4)}\right] & =\phi \phi_{y}(y-v)-\lambda_{4}(y-v)^{2} \phi \\
L_{2 k}\left[\varphi^{(5)}\right] & =-\phi \phi_{y}(y-v)-\lambda_{5}(y-v)^{2} \phi
\end{aligned}
$$

Application of the Fredholm alternative theorem yields the following expressions for the $\lambda_{i}$ :

$$
\begin{aligned}
& \lambda_{1}=\frac{\int_{0}^{1}\left(\operatorname{Ri} \theta^{2}+\phi_{y} \theta\right)(y-v) d y}{\int_{0}^{1} \phi^{2}(y-v) d y} \\
& \lambda_{2}=\frac{\int_{0}^{1} \phi^{2} d y}{\int_{0}^{1} \phi^{2}(y-v) d y} \\
& \lambda_{3}=-\left(R i \int_{0}^{1} \theta(2 k)\left(\theta_{y} \phi-\theta \phi_{y}\right) d y\right. \\
& \left.+\int_{0}^{1} \theta(2 k)(y-v)\left[\phi \phi_{y y}-\phi_{y}^{2}\right]_{y} d y\right) \\
& \left(\int_{0}^{1} \theta(2 k) \phi(y-v)^{2} d y\right)^{-1} \\
& \lambda_{4}=\frac{\int_{0}^{1} \theta(2 k) \phi \phi_{y}(y-v) d y}{\int_{0}^{1} \theta(2 k) \phi(y-v)^{2} d y} \\
& \lambda_{5}=-\lambda_{4}
\end{aligned}
$$

Figure 5 shows an example calculation of the phase velocity $v(k)$ for $R i=10$, and the eigenfunction $\varphi(y)$ with $k=0.05$. The two point boundary value problem (30) was solved numerically with homogeneous boundary conditions. As the boundary value problem is derived from an inviscid model, it does not suffer from the well known parasitic growth problem, thus standard numerical integration techniques (e.g. Runge-Kutta) are sufficient to maintain accuracy. The inviscid modes were solved for previously by Davey and Reid (1977) using a long wave limit. The extension to finite wavenumber is straightforward here. The only particular difficulty, somewhat apparent from Fig. 5, is that the approach of the eigenfunction to $y=1$ becomes steep as $R i \rightarrow 1 / 4$, 

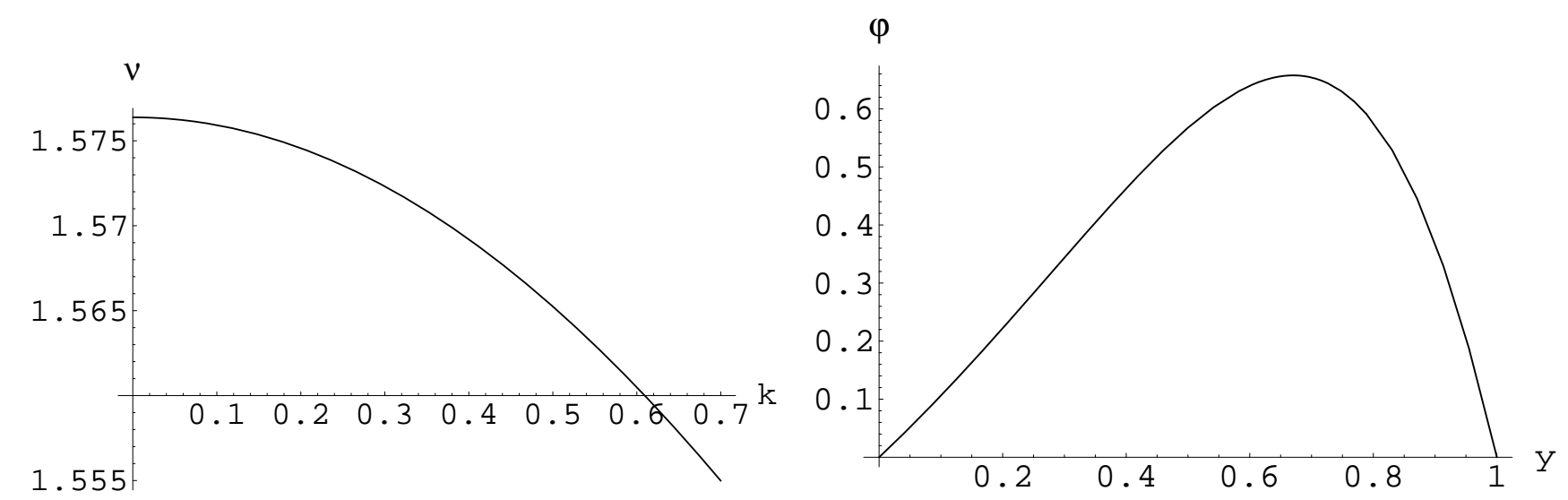

Fig. 5. (a) Phase velocity $v$ vs. wavenumber $k$ at $R i=10$. (b) Eigenfunction $\varphi$ vs. height $y$ with $k=0.05$ and $R i=10$.

causing Newton's method to fail. The bisection method, however, does not suffer from numerical difficulties in this case. The search for the phase velocity $v(k)$ was limited to $v>1$ in all cases.

There are two types of modes for the boundary value problem (19). Regular modes have $v_{n}>y$ for $y \in[0,1]$. Singular modes have $v_{n}=y_{\text {crit }}$ for a critical height $y_{\text {crit. }}$. The analysis given here tacitly assumes that $v_{n}$ is positive, i.e. the waves move rightward. For regular modes, it is clear that the denominator in Eq. (32) is always negative, regardless of $k$ and $R i$. For the singular modes it appears possible that the denominator can change signs. It can be verified that for the eigenfunctions found by Maslowe and Redekopp (1980) for the singular limit $k=0$ and Couette shearing, the denominator is also negative for all $R i$.

Figure 5 demonstrates weak dependence of $v(k)$ on $k-$ apparently quadratic with small coefficient $\sim-0.01$ in the long wave regime. The apparent quadratic in $k$ behaviour of $v(k)$ to a first approximation is entirely consistent with the assumptions of the long wave theory of Zimmerman and Velarde (1997). Figure 6 demonstrates the computation of the the $k$-dependence of the $\lambda_{i}$. Again, consistent with Zimmerman and Velarde (1997), the coefficients of the linear terms, $\lambda_{1}$ and $\lambda_{2}$, show a quadratic dependence in $k$ that is numerically small $(5-10 \%)$ of the leading order value. The coefficients of the nonlinear terms, $\lambda_{3}$ and $\lambda_{4}$, also demonstrate quadratic dependence on $k$.

Given that all the coefficients derived above for the separation condition demonstrate quadratic dependence on $k$ (least squared fits show the linear term in $k$ is of the order of truncation error), the whole of the long wave regime can be described by the $k \rightarrow 0$ leading order term and the coefficient of $O\left(k^{2}\right)$ for $v$ and the $\lambda_{i}$ for fixed $R i$. Representative values of $R i$ are taken in Table 1 and the coefficients of the power series expansion shown to $O\left(k^{2}\right)$. There are some key observations: (i) the higher order nonlinearity $\left(\lambda_{4}\right)$ is $2-3$ orders of magnitude smaller than the leading order nonlinearity $\left(\lambda_{3}\right)$ throughout this whole range; although the implication is that higher order nonlinearity is negligible, it turns out that it plays an important role in solitary wave collisions; (ii) the coefficient of the accumulation term $\lambda_{1}$ is practically invariant with $R i$; (iii) the coefficient $\lambda_{2}$ of the mixed derivative term $A_{\xi \xi \tau}$ does not become negligible within a representative range of $R i$, and is typically $O(1)$; (iv) formally, in order for the perturbation theory applied here to be valid, all of the terms $\varepsilon \lambda_{i}$ must be small relative to unity, putting an upper limit on the magnitude $\varepsilon$ of the wave amplitude; thus the formal constraint at low $R i$ is on the coefficient of nonlinearity $\varepsilon \lambda_{3} \ll 1$, and for high $R i$ it is on the accumulation coefficient $\varepsilon \lambda_{1} \ll 1$. In practice, however, the stability of the solitary wave depends on a balance between the leading nonlinear term and dispersion. Zimmerman and Velarde (1997) argue that the balance of these terms leads to the requirement that $U r^{*}=\varepsilon \lambda_{3} / k^{2} \approx 1$ so that solitary waves neither break by steepening nor by spreading. This condition permits extremely large solitary waves when $R i \gg 1$, since the numerically small coefficient $\lambda_{3}$ offsets large $\varepsilon$ values so that the product still satisfies the formal requirement of the perturbation theory. In this paper, however, the formal requirement would be violated in the high $R i$ regime as $\varepsilon \lambda_{1}$ becomes the limiting contribution.

\section{Solutions to the nonlinear evolution equation}

\subsection{Analytic solutions to the hybrid BBM-KdV equation}

In this section, the solutions to the separation (22) are considered. In light of the numerical values of $\lambda_{4} \ll \lambda_{3}$ for all $R i$, it is convenient to begin the discussion by neglecting the higher order nonlinear terms below. When numerical solutions are considered, however, the full Eq. (22), suitably scaled and transformed, will be simulated.

$k^{2} A_{\xi}+A_{\xi \xi \xi}+\varepsilon\left(\lambda_{1} A_{\tau}+\lambda_{2} A_{\xi \xi \tau}+\lambda_{3} A A_{\xi}\right)=0$

Equation (33) is an ordinary differential equation for the waveform in frame of $\xi=x-v t$. Regardless of the values of the coefficients (unless zero), this equation is equivalent to the $\mathrm{KdV}$ equation at steady state and always has 

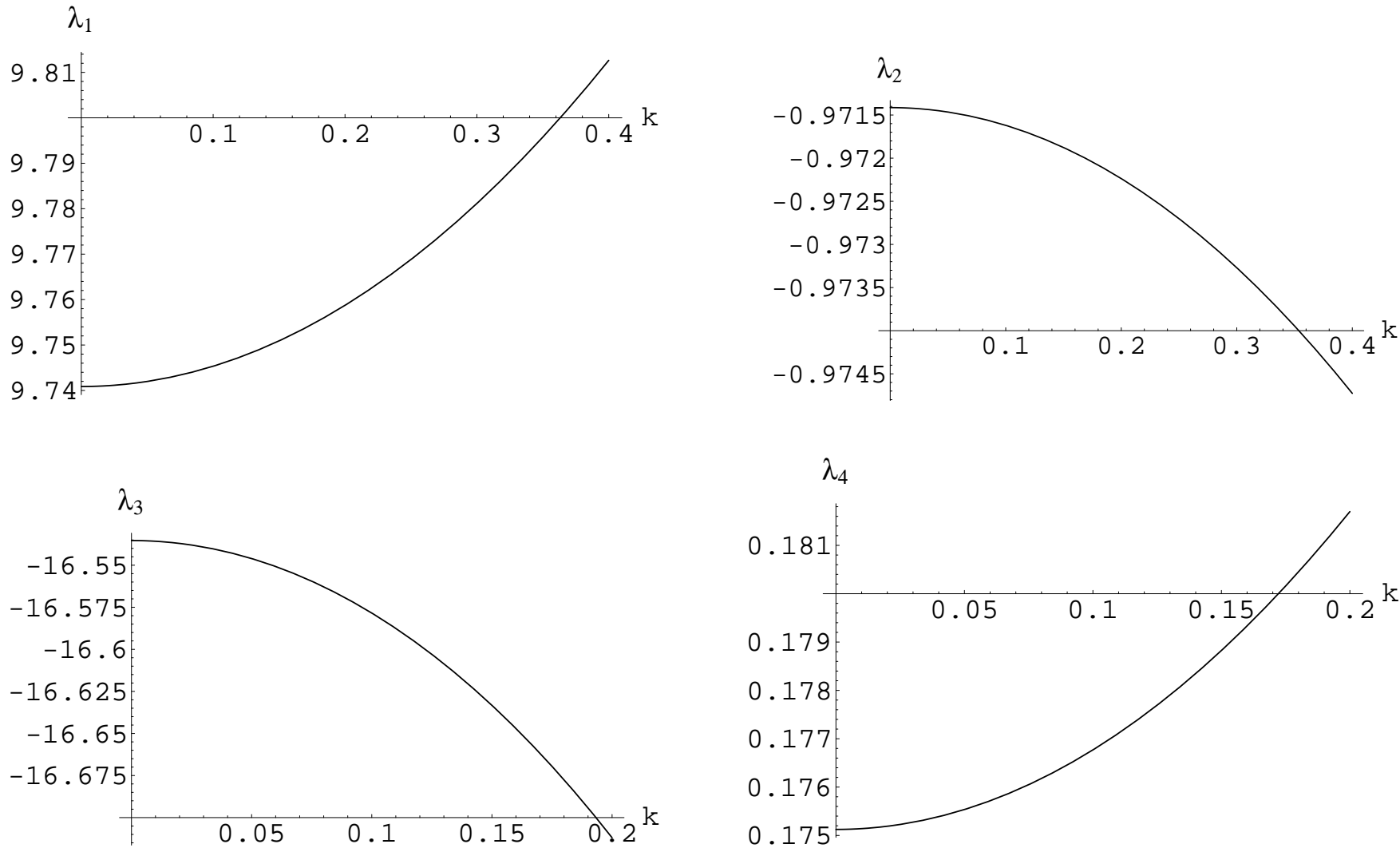

Fig. 6. Coefficients $\lambda_{1}, \lambda_{2}, \lambda_{3}, \lambda_{4}$ vs. wavenumber $k$ at $R i=10$.

Table 1. Representative values of the coefficients in Eq. (33) for various $R i$.

\begin{tabular}{rcccccccccc}
\hline \multicolumn{1}{r}{$R i$} & $c_{0}$ & $\left.\frac{\partial^{2} v}{\partial k^{2}}\right|_{k=0}$ & $\lambda_{1}$ & $\left.\frac{\partial^{2} \lambda_{1}}{\partial k^{2}}\right|_{k=0}$ & $\lambda_{2}$ & $\left.\frac{\partial^{2} \lambda_{2}}{\partial k^{2}}\right|_{k=0}$ & $\lambda_{3}$ & $\left.\frac{\partial^{2} \lambda_{3}}{\partial k^{2}}\right|_{k=0}$ & $\lambda_{4}$ & $\left.\frac{\partial^{2} \lambda_{4}}{\partial k^{2}}\right|_{k=0}$ \\
\hline 2. & 1.103 & -0.01199 & 8.960 & 1.012 & -1.963 & -0.09875 & -95.10 & -52.69 & 1.777 \\
5. & 1.310 & -0.02787 & 9.469 & 1.004 & -1.334 & -0.06638 & -31.73 & -11.32 & 0.4493 & 0.4358 \\
10. & 1.576 & -0.04444 & 9.662 & 1.000 & -0.9675 & -0.04846 & -16.54 & -4.547 & 0.1751 & 0.1648 \\
20. & 1.973 & -0.06665 & 9.764 & 1.000 & -0.6932 & -0.03487 & -9.445 & -2.148 & 0.07270 & 0.06678 \\
50. & 2.782 & -0.1091 & 9.827 & 1.000 & -0.4419 & -0.02230 & -4.942 & -0.9530 & 0.02445 & 0.02201 \\
100. & 3.705 & -0.1561 & 9.848 & 1.000 & -0.3133 & -0.01583 & -3.175 & -0.5670 & 0.01117 & 0.00995 \\
500. & 7.628 & -0.3523 & 9.865 & 1.000 & -0.1404 & -0.00710 & -1.248 & -0.2030 & 0.00197 & 0.00173 \\
1000. & 10.57 & -0.4987 & 9.867 & 1. & -0.09932 & -0.005022 & -0.8560 & -0.1352 & 0.00096 & 0.00084 \\
\hline
\end{tabular}

localised $\operatorname{sech}^{2}$ soliton solutions and periodic conoidal wave solutions. Equation (33) has the same form as the inviscid limit of a wave evolution equation found by a center manifold approach by Zimmerman and Velarde (1996) which approximates the vertical modal structure of temperature by a sine function and absorbs the error into the coefficients of the horizontal wave equation upon depth averaging.

In addition to the steady wave $\operatorname{sech}^{2}$ soliton solutions for Eq. (33), as the underlying dynamical system is dissipationfree and nonlinear, one must entertain the possibility of mul- tiple steady wave homoclinic solutions whose phase velocity depends on amplitude. It is logical to seek solutions in the form of Eq. (2):

$A=\operatorname{sech}^{2}\left(\frac{\xi-C t}{\Lambda}\right)$

Further, it is convenient to return to the original time scale:

$k^{2} A_{\xi}+A_{\xi \xi \xi}+\lambda_{1} A_{t}+\lambda_{2} A_{\xi \xi t}+\varepsilon \lambda_{3} A A_{\xi}=0$ 
Imposing Eq. (34) as solution to Eq. (35) yields the following constraints on $\Lambda$ and $C$ :

$$
\begin{aligned}
& \Lambda^{2}=8 \frac{1-C \lambda_{2}}{k^{2}-C \lambda_{1}+\varepsilon \lambda_{3}} \\
& C=\frac{-20+k^{2} \Lambda^{2}+2 \varepsilon \Lambda^{2} \lambda_{3}}{\Lambda^{2} \lambda_{1}-20 \lambda_{2}}
\end{aligned}
$$

That there is a solution of this form is not surprising. Equation (35) is a hybrid between the KdV equation and the similar BBM equation proposed for surface water waves by Peregrine (1966) and Benjamin et al. (1972). The BBM equation has been extensively studied by numerical integration (Bona, 1980) and shown to have $\operatorname{sech}^{2}$ solutions with collision properties that differ from the soliton (see Bona, 1980). The following remarks follow from Eq. (36):

- Since $\tau=\varepsilon t$, using the original time variable keeps the phase velocity $C$ as a regular quantity in $\varepsilon$.

- There is a double parameter $(\varepsilon, k)$ infinity of solutions with $\Lambda^{2}(\varepsilon, k)$ and $C(\varepsilon, k)$.

- Equation (36) has two solutions:

$$
\begin{array}{ll}
C=\frac{k^{2}}{\lambda_{1}}+\frac{\varepsilon \lambda_{3}}{3 \lambda_{1}}, & \Lambda^{2}=\frac{12 \lambda_{1}-12 k^{2} \lambda_{2}-4 \varepsilon \lambda_{2} \lambda_{3}}{\varepsilon \lambda_{1} \lambda_{3}} \\
C=\frac{1}{\lambda_{2}}, & \Lambda^{2}=0
\end{array}
$$

As the latter has apparently no physical meaning, it is rejected as extraneous. The former, however, has several meaningful special cases.

Classical solution theory for the $\mathrm{KdV}$ shows that the phase velocity $C$ is linearly proportional to the amplitude $\varepsilon$, and that the halfwidth is inversely proportional to the square root of the amplitude. This can be seen to hold in the same small amplitude, long wave limit for which Benney (1966) first derived the KdV equation for internal gravity waves. For $R i=10$ and small $k$ and $\varepsilon$, the asymptotic expansion of Eq. (37) yields

$$
\begin{aligned}
& C=0.1035 k^{2}+\left(-0.5705-0.09778 k^{2}\right) \varepsilon+O\left(k^{4}, \varepsilon^{2}\right) \\
& \Lambda^{2}=\frac{12}{\left(-16.54-4.547 k^{2}\right) \varepsilon}
\end{aligned}
$$

- Special case of no $\tau$-dependence, equivalent to $\lambda_{1}=\lambda_{2}=0$

This results in

$$
C=0 \quad \Lambda^{2}=\frac{8}{k^{2}+\varepsilon \lambda_{3}} \quad \varepsilon \rightarrow \frac{-3 k^{2}}{\lambda_{3}}
$$

The latter condition shows that there are only steady wave solutions in the reference frame $v$ if the constraint on the amplitude balancing weak dispersion is satisfied.
This is effectively a modified Ursell number for the situation:

$\frac{\varepsilon}{k^{2}}=\frac{-3}{\lambda_{3}}$

If $\mathcal{L}^{2}>0$, by the argument of the Introduction, $k=\mathcal{L}$ is the wavenumber selected by the waveguide for the longest wave trapped, then the amplitude of such waves is completely fixed by the Ursell number condition. Zimmerman and Velarde (1997) estimate $\lambda_{3}$ in the long wave limit as follows:

$\lambda_{3}=\frac{\lambda c}{(R i+2)|c-1|^{1 / 2}}\left(1-\left|\frac{c-1}{c}\right|^{3 / 2}\right)$,

where $\lambda=\sqrt{R i-\frac{1}{4}}$. This function becomes unbounded as $R i \downarrow 1 / 4$, demonstrating that this $\tau$-invariant steady wave has vanishing amplitude for strongly sheared, but stable stratifications. Conversely, this function vanishes as $R i \rightarrow \infty$, implying that this solitary wave is required to become extremely large as the stratification becomes strong. There is no violation of the formal requirements under which the weakly nonlinear theory was derived, as the nonlinear terms remain small both in Eq. (33) and the full heat and momentum equations. It should be noted that qualitatively, the predictions of solitary waves of temperature depression, the wavelength appropriate to the Scorer parameter, and a large amplitude disturbance occurring in a high $R i \approx 800$ waveguide are borne out in the STABLE wave event on 6 June 1986 reported here.

- Special case of the long wave limit $k=0$

It is noted that the halfwidth $\Lambda$ is given by

$\Lambda^{2} \rightarrow \frac{4\left(\frac{\lambda_{2}}{\lambda_{1}}+\frac{7}{\varepsilon \lambda_{3}}\right)}{3}$

with phase velocity

$C \rightarrow \frac{-\left(\varepsilon \lambda_{3}\right)}{7 \lambda_{1}}$

specified as the infinitely long wave limiting solution. With $\varepsilon \ll 1$, the former specifies an Ursell number

$\varepsilon \Lambda^{2} \rightarrow \frac{28}{3 \lambda_{3}}$

which agrees with the findings of Zimmerman and Velarde (1997) in the long wave limit. Thus, when $\mathcal{L}^{2}<0$, the expectation is that the longest trapped harmonic waves would be $k=0$, giving rise to the usual constraint that the nonlinearity balances dispersion so as to constrain the amplitude and halfwidth of the solitary wave jointly. 
It was found from the numerical evaluation of the coefficients $\lambda_{n}$ that at all $R i$, there is only a weak dependence on wavenumber $k$ (see Table 1), and thus the long wave limit $k=0$ is a good estimate for the coefficients appropriate to long waves. Thus, we can use Eq. (40) to test our original hypothesis that the $\mathcal{L}$ wavenumber is a good predictor of the halfwidth of a solitary wave of permanent form. We argue as follows. For the case of Couette shear, the definition (4) of the Scorer parameter $\mathcal{L}$, in the scales chosen here, reduces to

$$
\mathcal{L}^{2}=\frac{R i}{(y-v)^{2}}
$$

Consequently, the minimum wavenumber that does not radiate vertically is found at the bottom of the waveguide $y=0$ :

$\mathcal{L}_{m}^{2}=\frac{R i}{v^{2}}$

Equation (42) implies that for a solitary wave of permanent form, $\Lambda^{2} \sim 1 / \lambda_{3}$. Thus, if the halfwidth is predicted by the extreme Scorer parameter, then $\lambda_{3} v^{2} / R i$ must be a constant. Figure 7 tests this hypothesis. Strikingly, the desired near constant behaviour is found for high $R i$. This is gratifyingly in agreement with the observation from the STABLE data set illustrated in Fig. 3. The spike in $R i$ to $\sim 1000$ at about $10 \mathrm{~m}$ in height coincides with the extreme Scorer parameter which best approximates the halfwidth of the observed solitary wave of temperature depression shown in Fig. 1. Remarkably, the nonlinear theory derived here shows an intrinsic feature (the halfwidth $\Lambda$ ) of the nonlinear solitary wave that is set by the Scorer parameter of linear theory. One cannot help but wonder how this arises. A key point is that the solitary wave is of permanent form (34), which translates into a requirement on the modified Ursell number (42) that it be fixed at a value that balances nonlinearity and dispersion. So although the mathematical analysis permits the amplitude $\varepsilon$ to be a free parameter, the physical stability of the waveform (34) which requires the balance of nonlinearity and dispersion, is equivalent to fixing the value of the modified Ursell number (42). Since at high $R i$, the factor $\Lambda^{2} \sim 1 / \lambda_{3}$ is roughly constant, a fixed modified Ursell number imposes a fixed $\varepsilon$. Thus, in high $R i$ waveguides, the halfwidth and amplitude of solitary waves of permanent form are selected by the dynamics of the NEE and the stability criterion that the wave be of permanent form.

In the Introduction, the appropriateness of the modelling of the boundary conditions (10) used by both Benney and Benjamin was discussed. It should be clear at this point that the feature that is being modelled is the trapping of wave energy within a waveguide. As a fluid stratum embedded within a stratification, the vertical extent of the waveguide must itself be determined. Scorer's criterion (Scorer, 1949) for the trapping of wave energy essentially identifies the waveguide for which the boundary conditions (10) are a plausible model. The frequent recurrence of the halfwidth of observed solitary waves in agreement with the Scorer parameter (Rees and Rottman, 1994) is a consequence of the longevity of large amplitude solitary waves in high $R i$ waveguides.

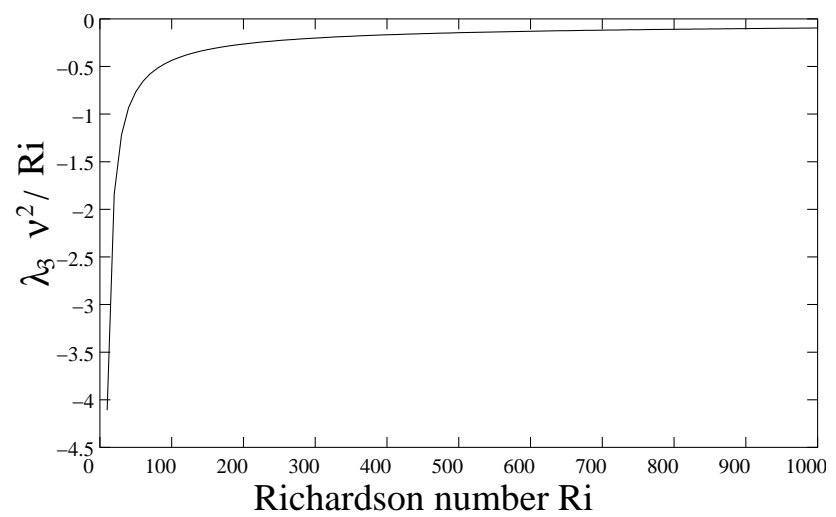

Fig. 7. Plot of $\lambda_{3} v^{2} / R i$ vs. $R i$. If the Scorer wavenumber $\mathcal{L}$ is inversely proportional to the halfwidth $\lambda$ of the solitary wave, then the quantity plotted on the ordinate should be constant with $R i$. It apparently approaches a constant at high $R i$.

The theory presented here predicts this occurrence in high $R i$ waveguides. Curiously, although the NEE derived here nonlinearizes about finite $k$ harmonic wave packets, this result about wavenumber selection is not sensitive to the basic wavenumber $k$, and holds in the $k=0$ limit. Thus, this prediction should be available from the theory derived from the infinitely long wave limit by either Benney or Benjamin. Nevertheless, in this paper, a new NEE which can be limited to finite wavenumber disturbances has been derived, so its properties should be characterized. In the next subsection, localized analytic solutions are presented. In the following section, numerical simulations explore the soliton-like collision properties of solitary wave solutions to the NEE.

\subsection{Analytic solutions of the nonlinear evolution equation}

Since the solitary wave form (34) fits solutions to the KdV equation and the BBM-KdV Eq. (33), it is reasonable to search for solutions of this form to the the full NEE (22). Surprisingly, the extra nonlinearity in this equation does not destroy the existence of an analytic solution of the homoclinic type, without recourse to further approximations. Imposing Eq. (34) as solution to the full nonlinear evolution Eq. (22) yields the following constraints on $\Lambda$ and $C$ :

$$
\begin{aligned}
& C=\frac{-20+k^{2} \Lambda+2 \Lambda \varepsilon \lambda_{3}-18 \varepsilon \lambda_{4}}{\Lambda \lambda_{1}-20 \lambda_{2}} \\
& \Lambda=\frac{8-8 C \lambda_{2}+6 \varepsilon \lambda_{4}}{k^{2}-C \lambda_{1}+\varepsilon \lambda_{3}}
\end{aligned}
$$

These constraints can be analytically solved for two solutions; only the following is physically meaningful: 
Table 2. Characterization of the solitary solutions (34) to the hybrid BBM-KdV Eq. (35), where $\lambda_{4}=0$, and ZR (22) equations for $R i=10$ and various $k, \varepsilon$ pairs.

\begin{tabular}{crcccc}
\hline$k$ & $\varepsilon$ & $C$ BBM-KdV & $\Lambda^{2}$ BBM-KdV & \multicolumn{1}{c}{$C$ ZR } & $\Lambda^{2} \mathrm{ZR}$ \\
\hline 0.05 & -1 & 0.570442 & 0.725191 & 0.5336 & 0.972316 \\
0.05 & -6 & 3.42446 & 0.120865 & 2.72698 & 0.312483 \\
0.05 & -12 & 6.84866 & 0.0604326 & 4.82226 & 0.215112 \\
\hline
\end{tabular}

$$
\begin{aligned}
C & =\frac{-3 k^{2}\left(1+\varepsilon \lambda_{4}\right)+\varepsilon \lambda_{3}\left(4+3 \varepsilon \lambda_{4}\right)}{D} \\
& +\frac{\left(k^{2}+2 \varepsilon \lambda_{3}\right)}{\lambda_{1} D}\left(\lambda_{2}\left(3 k^{2}+\varepsilon \lambda_{3}\right)\right)+\frac{\left(k^{2}+2 \varepsilon \lambda_{3}\right)}{\lambda_{1} D} \\
& \times \sqrt{-6 \varepsilon^{2} \lambda_{1} \lambda_{2} \lambda_{3} \lambda_{4}+\left(\lambda_{2}\left(3 k^{2}+\varepsilon \lambda_{3}\right)-3 \lambda_{1}\left(1+\varepsilon \lambda_{4}\right)\right)^{2}} \\
\Lambda^{2} & =\frac{-2 \lambda_{2}\left(3 k^{2}+\varepsilon \lambda_{3}\right)}{\varepsilon \lambda_{1} \lambda_{3}}+\frac{3\left(1+\varepsilon \lambda_{4}\right)}{\varepsilon \lambda_{3}}+\frac{2}{\varepsilon \lambda_{1} \lambda_{3}} \\
& \times \sqrt{-6 \varepsilon^{2} \lambda_{1} \lambda_{2} \lambda_{3} \lambda_{4}+\left(\lambda_{2}\left(3 k^{2}+\varepsilon \lambda_{3}\right)-3 \lambda_{1}\left(1+\varepsilon \lambda_{4}\right)\right)^{2}}
\end{aligned}
$$

where

$$
\begin{aligned}
& D=-\lambda_{2}\left(3 k^{2}+11 \varepsilon \lambda_{3}\right)+3 \lambda_{1}\left(1+\varepsilon \lambda_{4}\right) \\
& +\sqrt{-6 \varepsilon^{2} \lambda_{1} \lambda_{2} \lambda_{3} \lambda_{4}+\left(\lambda_{2}\left(3 k^{2}+\varepsilon \lambda_{3}\right)-3 \lambda_{1}\left(1+\varepsilon \lambda_{4}\right)\right)^{2}}
\end{aligned}
$$

For $R i=10$ and small $k$ and $\varepsilon$, the asymptotic expansion of Eq. (46) yields

$$
\begin{aligned}
& C=0.1035 k^{2}+\left(-0.5705-0.09778 k^{2}\right) \varepsilon+O\left(k^{4}, \varepsilon^{2}\right) \\
& \Lambda^{2}=0.2734+k^{2}\left(-0.1061+\frac{0.1269}{\varepsilon}\right)-\frac{0.7257}{\varepsilon}+O\left(k^{4}, \varepsilon\right)(47)
\end{aligned}
$$

This is closely comparable with the approximation of $\lambda_{4}=0$ of the last subsection. As it has just been shown that there is a new, analytic solution of the solitary wave form to a newly derived inviscid NEE, it is conventional to explore by numerical simulations along the lines of the seminal paper by Zabusky and Kruskal (1965) whether the solutions are soliton-like in character.

Table 2 demonstrates the distinctly different solitary wave features for the two different solitary wave equations explored here - the ZR solitary wave is always broader and slower propagating due to the presence of the hypernonlinearity with coefficient $\lambda_{4}$.

\subsection{Numerical simulations of the nonlinear evolution equation}

It is well known that NEEs that are modified KdV equations are difficult to simulate numerically. For instance the $\mathrm{KdV}$ KSV equation with both instability and hyperdiffusion terms required treatment by the method of variational embedding
(Christov and Velarde, 1995), as standard finite difference approximations were found to be inherently unstable.

One technique that has demonstrated robust stability properties is the method of lines (MOL). Schiesser (2001) describes the salient features that the spatial discretization is established permanently in the simulation, but the temporal solution is adaptive, using local error estimates to adapt the time step to within the desired error tolerance. We coded the method of lines, using Runge-Kutta-Fehlberg (a 4-5 method) in time, and up to seven point differences to accomodate the $A_{\xi \xi \xi}$ term. Figure 8 shows the evolution of a single localized disturbance under the approximate $\mathrm{KdV}$ dynamics and of the ad hoc removal of the "BBM term" $A_{\xi \xi t}$, which has only the extra nonlinearity in addition to the KdV terms. Although this method proved adequate for the approximations attempted in Fig. 8, demonstrating the known analytic form of solution Eq. (34), it was not possible to include either the BBM term nor to resolve overtaking collisions of two initial disturbances. With the method of lines technique, the BBM term, $A_{\xi \xi t}$, can only be included consistently by finite difference approximations at the last time step. This delay contributes to numerical instability, which manifests as the inability to adapt the time step to the error tolerance. The collision simulation with MOL performs adequately with the $\mathrm{KdV}$ equation, with nonlinearity treated by delayed differences. However, the additional nonlinear terms form a shock wave upon collision which cannot be resolved by the technique.

Since the difficulty lies within the delayed difference treatment of some terms, the prescribed solution is to use semiimplicit integration schemes. The workhorse method is the Crank-Nicholson scheme, which uses spatial centered differences arithmetically averaged between the current time and the future time step. These must be simultaneously solved. In the method adopted here, the linear terms are treated in the Crank-Nicholson fashion. The nonlinear terms are approximated by delays. The BBM term is treated by an Euler step in time, centered difference at both the current and future time. If $A(\xi=j h, \tau=n \Delta t)=A_{j}^{n}$, where $h$ is the spatial resolution, then a banded matrix equation results:

$$
\begin{aligned}
& a_{1} A_{j+2}^{n+1}+a_{2} A_{j+1}^{n+1}+a_{3} A_{j}^{n+1}+a_{4} A_{j-1}^{n+1}+a_{5} A_{j-2}^{n+1} \\
& \quad=b_{1} A_{j+2}^{n}+b_{2} A_{j+1}^{n}+b_{3} A_{j}^{n}+b_{4} A_{j-1}^{n}+b_{5} A_{j-2}^{n} \\
& \quad+N\left(A, A_{\xi}, A_{\xi \xi}, A_{\xi \xi \xi}\right)
\end{aligned}
$$



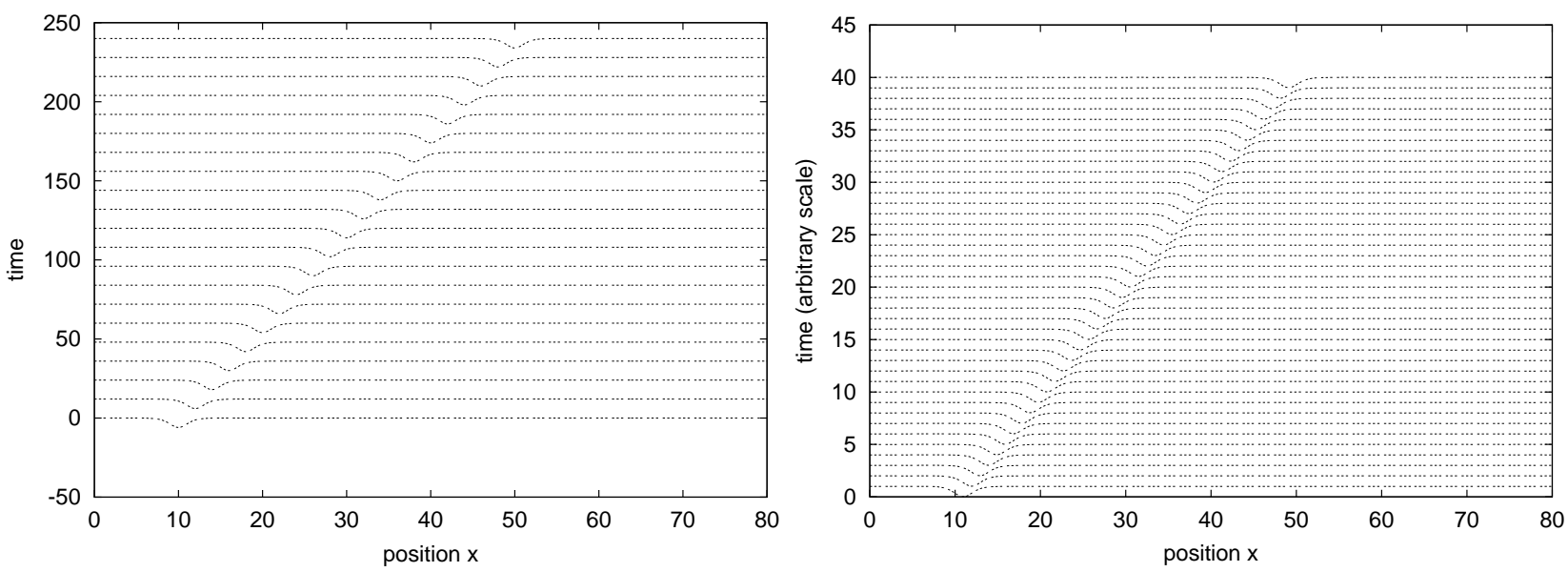

Fig. 8. (a) Simulation of only the KdV terms in Eq. (35) and (b) Simulation of the all terms in Eq. (35) except the BBM $A \xi \xi t$ term; for $R i=10$ and $k=0.05$, with an initial conditions of Eq. (34) with (a) $\varepsilon=-6$; (b) $\varepsilon=-1$ with origin shifted to $x_{0}=10$. Time is scaled arbitrarily so as to graphically separate successive time steps. In (a) $t \in[0,20]$. In (b), $t \in[0,40]$. Both simulations have spatial resolution $h=0.1$ horizontally.

The scheme is first order in time, second order in space. For the purposes of discussion, it is necessary to note only that $a_{1}=1 / 2 h^{3}$ and $a_{3}=\lambda_{1} / \Delta t-\lambda_{2} / \Delta t h^{2}$. CrankNicholson methods are absolutely stable (see e.g. Ganzha and Vorozhtsov (1996) for the von Neumann criterion). However, the sparse solver method becomes poorly conditioned if the matrix is not diagonally dominant, leading to spurious oscillations. Typically, for third order pdes, the condition for diagonal parity is that $\Delta t \approx h^{3}$, i.e. the first term of $a_{3}$ is of the same order as $a_{1}$. This necessitates ridiculously small times steps $\Delta t$ for any common choice of resolution in $\xi$, say $10^{-2}$. In this case, however, diagonal parity is achieved by balancing the second term of $a_{3}$ with $a_{1}$, yielding $\Delta t \approx h$. This is apparently a desirable situation from the standpoint of stability. Curiously, however, the system is poorly conditioned at achieving accuracy with decreasing $\Delta t$ and $h$. As the second term in $a_{3}$, which originates from the BBM term, always dominates the first term as $h \downarrow$, it is found that the linear terms in Eq. (48) approximate an identity operator on any signal $A(\xi)$ at a given time. ( $b_{3}$ has a similar structure, which leads to the mapping $A_{j}^{n+1} \leftarrow A_{j}^{n}$ as $h \downarrow$. Thus, only since $\lambda_{1}$ is naturally larger in modulus than $\lambda_{2}$ (see Table 1) is there a window in simulation parameter space $(h, \Delta t)$ which is reasonably accurate and numerically stable.

As an aside, it is possible to evade the whole issue of simulating the BBM $A_{\xi \xi \tau}$ term via applying the perturbation assumption. Peregrine (1966), in deriving the equation for an undular bore, noted that up to the order of the error in the perturbation scheme, $A_{x x t} \sim A_{x x x}$. In this case, the fundamental assumption of a harmonic wave to leading order Eq. (18), allows the approximation $\lambda_{2} A_{\xi \xi \tau} \sim-k^{2} \lambda_{2} A_{\tau}$, which is a minor modification to the accumulation term. However, this is formally only valid for $\varepsilon \ll 1$. Indeed, although the NEE derived here Eq. (35), is formally only valid for small $\varepsilon$, in the case of high $R i$ (see Table 1), the coefficients of the nonlin- ear terms are naturally small, implying that large amplitude is possible before the practical requirement that the neglected terms are appreciable comes into force. Consequently, a robust simulation for large $\varepsilon$ has practical value.

Figure 9 demonstrates the Crank-Nicholson simulation of the full NEE Eq. (35) for single and double localized disturbance initial conditions of the form Eq. (34) appropriate to the analytic solution for $C$ and $\Lambda$. In contrast to the MOL solutions, here the solitary wave amplitude is taken as intrinsically positive, even though $\varepsilon=-1$. So these frames both represent waves of temperature and streamfunction depression. Frame (a) agrees roughly with the expected analytically predicted phase velocity $C$ for the given conditions. Frame (b) demonstrates a clear phase shift - a boost to the larger, overtaking wave and a delay to the slower wave - due to the collision. The waves themselves appear unchanged at this resolution. These features establish the soliton-like character of the solutions (34) as originally established by Zabusky and Kruskal (1965).

Contemporary connotations of soliton character imply that the equation is integrable (e.g. Cervero and Zurron, 1996), and thus an inverse scattering transform exists (for instance, Gardner et al., 1967) which permits the characterization of the time asymptotic state. Whether or not the NEE (35) is integrable is not addressed here. There is greater likelihood that it is integrable if it conserves wave energy, which is tested below. Multiplying the NEE by $A$, and integrating over all space yields:

$$
\begin{aligned}
& \lambda_{1} \frac{d}{d t} \int_{-\infty}^{\infty} A^{2} d \xi=-\lambda_{2} I_{2}-k^{2} \int_{-\infty}^{\infty} A A_{\xi \xi \xi} d \xi \\
& \quad+\varepsilon \lambda_{3} \int_{-\infty}^{\infty} A^{2} A_{\xi} d \xi-\varepsilon \lambda_{4} I_{4}
\end{aligned}
$$



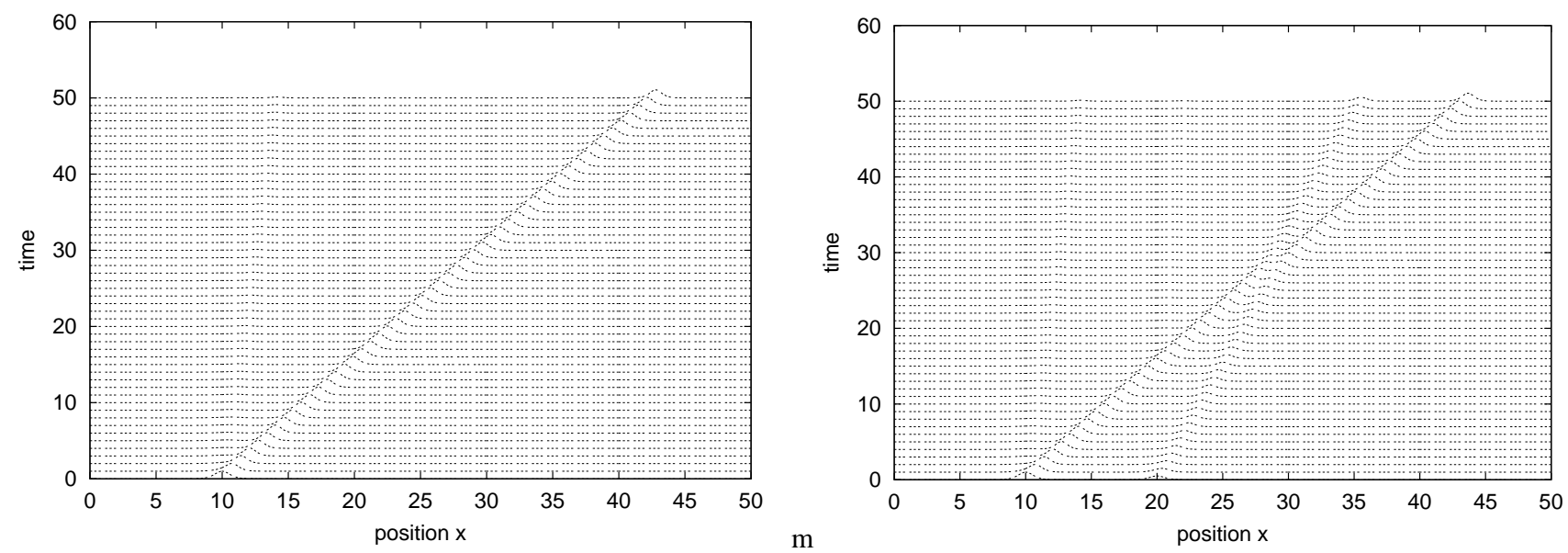

Fig. 9. Simulation of the full Eq. (35); for $R i=10$ and $k=0.05$, with an initial conditions of Eq. (34) with $\varepsilon=-1$, with origin shifted to (a) $x_{0}=10$ for a single disturbance of unit amplitude and (b) an additional disturbance initially at $x_{1}=20$ with half unit amplitude. In both figures, integer times and unit amplitudes are displayed. Both simulations have $h=0.025$ horizontally and $\Delta t=0.001$.

Terms two and three on the RHS of Eq. (49) are well known from the $\mathrm{KdV}$ equation to identically vanish with boundary conditions that $A$ and all its derivatives vanish at $\xi \rightarrow \pm \infty$. The other two terms will be considered individually, by integration by parts. Only the first contribution to $I_{4}$ need be considered, as one integration by parts yields the same form as the other term, which is constructively reinforced:

$$
\begin{aligned}
I_{4} & =\int_{-\infty}^{\infty}\left[A^{2} A_{\xi \xi \xi}-A A_{\xi} A_{\xi \xi}\right] d \xi \\
& =-3 \int_{-\infty}^{\infty} A\left(A_{\xi} A_{\xi \xi}\right) d \xi \\
& =-\frac{3}{2} \int_{-\infty}^{\infty} A \frac{\partial A_{\xi}^{2}}{\partial \xi} d \xi=\frac{3}{2} \int_{-\infty}^{\infty} A_{\xi}^{3} d \xi
\end{aligned}
$$

The second term is readily evaluated as

$$
I_{2}=\frac{d}{d \tau} \int_{-\infty}^{\infty} A A_{\xi \xi} d \xi=-\frac{d}{d \tau} \int_{-\infty}^{\infty} A_{\xi}^{2} d \xi
$$

The latter, combined with Eq. (49) leads to the conclusion that wave energy and momentum are not conserved, but rather are interchanged and can be created or destroyed, according to the sign of $A_{\xi}$ as seen below.

$$
\frac{d}{d \tau} \int_{-\infty}^{\infty}\left(\lambda_{1} A^{2}+\lambda_{2} A_{\xi}^{2}\right) d \xi=-\frac{3}{2} \varepsilon \lambda_{4} \int_{-\infty}^{\infty} A_{\xi}^{3} d \xi .
$$

Although it should be noted that the RHS Eq. (52) has a small constant of proportionality, for steep waveforms the NEE lacks any mechanism to oppose shock formation. Thus, dissipative mechanisms that were neglected in the model (viscous friction, thermal or solutal diffusivity) would play a necessary role in preventing the formation of a discontinuity through feedback from wave steepening through the RHS of Eq. (52). Otherwise, if $\lambda_{4}$ is negligible, it can be seen that the weighted combination of wave energy and wave momentum $\left\langle\lambda_{1} A^{2}+\lambda_{2} A_{\xi}^{2}\right\rangle$ is conserved. The angle brackets refer to the integral over all space. The NEE can be differentiated with respect to $\xi$, multiplied by $A$, and integrated over all space, which by analogy with the above Eq. (49), should yield a conservation of $\left\langle\lambda_{1} A_{\xi}^{2}+\lambda_{2} A_{\xi \xi}^{2}\right\rangle$. Iterating this process will lead to an infinity of conserved quantities. Whether this is sufficient to demonstrate that this system is integrable is an open question. If so, then there is likely to be an inverse scattering transformation, which would theoretically specify the time asymptotic state. Zimmerman and Haarlemmer (1999a) have demonstrated some utility in the discrete periodic inverse scattering transform (DPIST) for analysis of wave propagation in a stratified fluid. The DPIST analysis identified the "soliton" component of a wave passing the sensor that was unchanged at a second sensor downstream in a stratified wave tank. Osborne and coworkers (see Osborne and Petti, 1994, and references therein) have pioneered the use of DPIST as a data analysis tool for surface water wave data sets.

It should be noted that the NEE can be formally modified by application of the original separation condition that to leading order, the waveform should be a harmonic wave. This permits the formal substitution that $A_{\xi \xi \tau}=-k^{2} A_{\tau}+O(\varepsilon)$. This alters the conserved quantity to $\left\langle\left(\lambda_{1}-k^{2} \lambda_{2}\right) A^{2}\right\rangle$. It also reduces the NEE, with the assumption $\left|\lambda_{4}\right| \ll 1$, to the KdV equation. Thus, the NEE is a "nearly integrable" system, for which DPIST may prove sufficiently accurate for long waves of nearly permanent form. 


\section{Conclusions}

In this paper, the important role of the Scorer parameter in defining a waveguide that traps wave energy in a stratum embedded in a deeper fluid is argued. In particular, it is noted that infinitely long waves are not trapped horizontally in such a waveguide. Atmospheric observations illustrated the remarkable fact that in waveguides so defined, and characterized by a high $R i$, the halfwidth of observed large amplitude disturbances coincides with the wavenumber of the Scorer parameter. It is remarkable that an intrinsic feature of a nonlinear wave is dependent on a feature of the linear theory, especially since canonical $\mathrm{KdV}$ theory would predict that key features of a solitary wave, halfwidth and phase velocity, are amplitude dependent. Thus a new theory, not limited to infinitely long waves as the basic state, is derived. For stably stratified fluid layers under Couette shear embedded in a deeper fluid, an expansion procedure is presented for weakly nonlinear, planar shallow water waves. Nonlinear wave equations are derived for basic states-travelling waves admitted by the linearized system-internal gravity waves modified by shear. Under the common approximation that buoyancy affects little the inertia of the wave, the coefficients of the evolution equations are uniquely determined as functionals of the shear and stratification profiles, for all $R i$ and $k$. Limiting cases are analyzed, demonstrating that in high $R i$ waveguides, solitary waves of permanent form do have halfwidths inversely proportional to the Scorer parameter. A localized analytic solution to the NEE for finite wavenumber basic disturbances is found.Numerical simulations explore the soliton-like collision behaviour of two such waves engaged in an overtaking collision. For values of the coefficients appropriate for $R i=10$ in linear shear, the initial states recur with a "phase shift". Whether this is a consequence of the "near integrability" of the NEE for these coefficients is discussed by energy arguments. If the smallest nonlinear terms are neglected, the NEE has an infinite number of conserved quantitities, so it may be integrable itself. Arguments consistent with its original derivation would make this approximate form for the NEE equivalent to the KdV equation, which is structurally "near integrable" if not integrable in its own right. The integrability is an important concern if an inverse scattering transform analysis, improving on the DPIST methodology applied by Osborne and coworkers (Osborne and Petti, 1994), among others, is to be developed based on this NEE.

Acknowledgements. The authors would like to thank the National Science Foundation and North Atlantic Treaty Organization for a postdoctoral fellowship (WBZ) and collaborative research grant, CRG 940242. JMR is grateful to the NERC for the provision of grant number GR3/09321. Helpful discussions with M. G. Velarde, J. C. King, P. Anderson, and G. W. Haarlemmer are acknowledged. We thank B. N. Hewakandamby for the use of his numerical simulation package. WBZ especially welcomed commentary by the late W. G. Pritchard and T. B. Benjamin on this topic. We would like to thank the British Antarctic Survey for allowing us to use data from their STABLE project.
Edited by: A. Osborne

Reviewed by: two referees

\section{References}

Benjamin, T. B.: Internal waves of finite amplitude and permanent form, J. Fluid Mech., 25, 241-270, 1966.

Benjamin, T. B., Bona, J. L., and Mahony, J. J.: Model equations for long waves in nonlinear dispersive systems, Philos. Trans. Roy. Soc. London, Ser. A 272, 47-78, 1972.

Benney, D. J.: Long nonlinear waves in fluid flows, J. Math. Phys., 45, 52-63, 1966.

Bona, J. L., Pritchard, W. G., and Scott, L. R.: Solitary-wave interaction, Phys. Fluids, 23(3), 438-441, 1980.

Bona, J. L., Pritchard, W. G., and Scott, L. R.: Numerical schemes for a model of nonlinear dispersive waves, J. Comp. Phys., 60(2), 167-186, 1985.

Boussinesq, J. V.: Theorie de l'intumescence liquide appelee onde solitaire ou de translation sepropageant dans un canal rectangulaire, C. R. Acad. Sci., 72, 755-759, 1871.

Boussinesq, J. V.: Theorie generale des mouvements qui sont propages dans un canal rectangulaire horizontal, C. R. Acad. Sci., 73, 256-260, 1871.

Boussinesq, J. V.: Theorie des ondes et des remous qui se propagent le long d'un canal rectangulaire horizontal en communiquant au liquid contenu dans ce canal des vitesses sensiblement pareilles de la surface au fond, J. Math. Pures Appl., 17, 55, 1872.

Cerveró, J. M. and Zurrón, O.: Integrability of the perturbed KdV equation for convecting fluids: symmetry analysis and solutions, Nonlin. Math. Phys., 3, 1-23, 1996.

Christov, C. I. and Velarde, M. G.: Dissipative solitons,Physica, D86, 323-347, 1995.

Davey, A. and Reid, W. H.: On the stability of stratified viscous plane Couette flow: Part 1, constant buoyancy frequency, J. Fluid Mech., 80(3), 509-525, 1977.

Doviak, R. J., Chen, S. S., and Christie, D. R.: A thunderstorm generated solitary wave observation compared with theoryfor nonlinear waves in a sheared atmosphere, J. Atmos. Sci., 48(1), 87$111,1991$.

Ganzha, V. G. and Vorozhtsov, E. V.: Numerical solutions for partial differential equations, CRC Press, Boca Raton, 1996.

Gardner, C. D., Greene, J. M., Kruskal, M. D., and Miura, R. M.: Method for solving the Korteweg-de Vries equation, Phys. Rev. Lett., 19, 1095-1097, 1967.

Gear, J. A. and Grimshaw, R.: A second-order theory for solitary waves in shallow fluids, Phys. Fluids, 26(1), 14-29, 1983.

Goldstein, S.: On the stability of superposed streams of fluids of different densities, Proc. Roy. Soc. A, 132, 524-548, 1931.

Hewakandamby, B. N. and Zimmerman, W. B.: Dynamics of surface spreading of insoluble surfactants on flowing thin films, Phys. Fluids, accepted, 2003.

King, J. C. and Anderson, P. S.: Installation and performance of the STABLE instrumentation at Halley, Br. Antarc. Survey Bull., 79, 65-77, 1988.

Koop, C. G. and Butler, G.: An investigation of internal solitary waves in a two-fluid system, J. Fluid Mech., 112, 225-251, 1981.

Lord Rayleigh: On waves, Philos. Mag., 1, 257-279, 1876.

Maslowe, S. A. and Redekopp, L. G.: Long nonlinear waves in stratified shear flows, J. Fluid Mech., 101(2), 321-348, 1980. 
Osborne, A. R. and Petti, M.: Laboratory-generated, shallow-water surface waves: Analysis using the periodic, inverse scattering transform, Phys. Fluids, 6(5), 1727-1744, 1994.

Peregrine, D. H.: Calculations of the development of an undular bore, J. Fluid Mech., 25, 321-330, 1966. .

Rees, J. M.: On the characteristics of eddies in the stable atmospheric boundary layer, Boundary-Layer Met., 55, 325-343, 1991.

Rees, J M. and Rottman, J. W.: Analysis of solitary disturbances over an antarctic ice shelf, Boundary-Layer Met., 69, 285-310, 1994.

Rees, J. M., Denholm-Price, J. C., King, J. C., and Anderson, P. S.: A climatological study of internal gravity waves in theatmospheric boundary layer overlying the Brunt Ice Shelf, Antarctica, J. Atmos. Sci., 57, 511-526, 2000.

Vande Wouwer, A., Saucez, P., and Schiesser, W. E.: Adaptive method of lines, Chapman \& Hall / CRC Press, 2001.

Scorer, R. S.: Theory of waves in the lee of mountains, Quart. J. R. Meteorol. Soc., 75, 41-56, 1949.
Taylor, G. I.: Effect of variation in density on the stability of superposed streams of fluid, Proc. Roy. Soc. A, 132, 499-523, 1931.

Ursell, F.: The long wave paradox in the theory of gravity waves, Proc. Camb. Phil. Soc., 49, 685-694, 1953.

Weidman, P. D. and Velarde, M. G.: Internal Solitary Waves, Studies in App. Math., 86, 167-184, 1992.

Zabusky, N. J. and Kruskal, M. D.: Interaction of "solitons" in a collisionless plasmaand the recurrence of initial states, Phys. Rev. Lett., 15, 240-3, 1965.

Zimmerman, W. B. and Haarlemmer, G. W.: Internal gravity waves: Analysis using the periodic, inverse scattering transform, Nonlin. Proc. Geophys., 6, 11-26, 1999.

Zimmerman, W. B. and Velarden, M. G.: A centre manifold approach to solitary internal waves in a sheared, stably stratified fluid layer, Nonlin. Proc. Geophys., 3, 110-114, 1996.

Zimmerman, W. B. and Velarde, M. G.: Strong dispersive effects on internal nonlinear waves in a sheared, stablystratified fluid layer, Wave Motion, 30, 159-173, 1999. 